\title{
The Application of a Special Hermite Finite Element Coupled with Collocation to the Diffusion Equation ${ }^{\star}$
}

\author{
Lidiya Gileva ${ }^{1[0000-0001-7926-6642]}$, Evgeniya Karepova ${ }^{1,2}$ [0000-0002-6515-2932], \\ and Vladimir Shaidurov ${ }^{1[0000-0002-7883-5804]}$ \\ 1 Institute of Computational Modelling of SB RAS, \\ 660036, Akademgorodok, Krasnoyarsk, Russia \\ gileva@icm.krasn.ru \\ e.d.karepova@icm.krasn.ru \\ shaidurov04@mail.ru \\ http://icm.krasn.ru \\ 2 Siberian Federal University, 79 Svobodny pr., 660041 Krasnoyarsk, Russia
}

\begin{abstract}
In the paper, we propose an efficient method based on the use of a bicubic Hermite finite element coupled with collocation for the diffusion equation. This enables one to reduce the dimension of the system of equations in comparison with the standard finite element scheme. Numerical experiments confirm a theoretical convergence estimate and demonstrate the advantage of the proposed method.
\end{abstract}

Keywords: Diffusion equation - Finite element method $\cdot$ Hermite finite element · Collocation method.

\section{Introduction}

The application of Hermite finite elements to the solution of a partial differential equation results in a system of linear algebraic equations whose dimension is several times less than in the case of full or incomplete (serendipity) Lagrange elements of the same degree which provide the same order of convergence [8]. Besides, a finite element with the values of second-order derivatives at the nodes in the set of degrees of freedom (DoF) provides a way to use the collocation technique for some equations. Requiring the residual to vanish at the nodes, we express one DoF in terms of other ones from the original equation and eliminate the related equations from the system. This enables one to reduce still further the dimension of the system of equations. In [4] we propose this approach for the Poisson equation and confirm its efficiency with numerical experiments.

In the paper, we extend this approach to the diffusion equation with a constant coefficient on a rectangular domain. To construct a discrete problem, we use the bicubic Hermite element on a rectangular cell proposed in [4] and eliminate the unknowns being the values of the second-order derivative with respect

\footnotetext{
* Supported by Project 17-01-00270 of Russian Foundation for Basic Research.
} 
to one of variables. We prove an error estimate for the modified problem. $\mathrm{Nu}$ merical experiments confirm that the elimination of $1 / 3$ of the unknowns and the equations retains the order of convergence of an approximate solution.

A direct application of Hermite elements on a rectangular cell is restricted to the case of domains composed of rectangles. However, to extend the area of the application of the bicubic Hermite element considered in the paper to the case of a polygonal domain or a domain with the curved boundary, we can complement it with the appropriate triangular elements proposed and studied in [5].

\section{Notations}

Let $\Omega=\left(0, H_{1}\right) \times\left(0, H_{2}\right) \subset R^{2}$ be a rectangle with the boundary $\Gamma$. To construct a uniform triangulation $\mathcal{T}_{h}$, we subdivide $\bar{\Omega}$ into $N_{1} \times N_{2}$ closed rectangles by the lines

$$
x=i h_{1}, \quad y=j h_{2}, \quad i=0, \ldots, N_{1}, \quad j=0, \ldots, N_{2},
$$

where $h_{k}=H_{k} / N_{k}, k=1,2$. Denote

$$
h=\max \left\{h_{1}, h_{2}\right\} .
$$

For functions $u, v$ defined and measurable on $\Omega$, introduce the inner product and the induced norm:

$$
(u, v)=\int_{\Omega} u v d \Omega, \quad|u|_{0, \Omega}=(u, u)^{1 / 2} .
$$

Denote by $L_{2}(\Omega)$ the space of all functions $u$ defined and measurable on $\Omega$ with the finite norm $|u|_{0, \Omega}$. Introduce also the semi-norms

$$
|u|_{k, \Omega}=\left(\sum_{i_{1}+i_{2}=k}\left|\frac{\partial^{k} u}{\partial x^{i_{1}} \partial y^{i_{2}}}\right|_{0, \Omega}^{2}\right)^{1 / 2} .
$$

Denote by $H^{k}(\Omega)$ the space of all functions $u$ defined and measurable on $\Omega$ together with their partial derivatives up to order $k$ with the finite norm [1]

$$
\|u\|_{k, \Omega}=\left(|u|_{0, \Omega}^{2}+|u|_{1, \Omega}^{2}+\ldots+|u|_{k, \Omega}^{2}\right)^{1 / 2} .
$$

Denote by $P_{k}$ the space of all polynomials in two variables up to degree $k$ :

$$
\sum_{0 \leq i_{1}+i_{2} \leq k} a_{i_{1}, i_{2}} x^{i_{1}} y^{i_{2}}
$$

Define a "reference" finite elements as a triple $(\hat{e}, \hat{P}, \hat{\Sigma})$ where $\hat{e}$ is a "reference" cell, $\hat{P}$ is a space of polynomials defined on $\hat{e}$, and $\hat{\Sigma}$ is a set of functionals called degrees of freedom. 


\section{A Bicubic Hermite Element}

In [4] we propose a new Hermite element defined as follows (Fig. 1):

$$
\begin{gathered}
\hat{e}=[0,1]^{2}, \\
\hat{P}=\operatorname{span}\left\{1, \hat{x}, \hat{y}, \hat{x} \hat{y}, \hat{x}^{2}, \hat{y}^{2}, \hat{x}^{2} \hat{y}, \hat{x} \hat{y}^{2}, \hat{x}^{3}, \hat{y}^{3}, \hat{x}^{3} \hat{y}, \hat{x} \hat{y}^{3}\right\}, \\
\hat{\Sigma}=\left\{\hat{\psi}_{s, i}(s=0,1,2): \hat{\psi}_{0, i}(p)=p\left(\hat{a}_{i}\right), \hat{\psi}_{1, i}(p)=\partial^{2} p / \partial \hat{x}^{2}\left(\hat{a}_{i}\right),\right. \\
\left.\quad \hat{\psi}_{2, i}(p)=\partial^{2} p / \partial \hat{y}^{2}\left(\hat{a}_{i}\right), i=1, \ldots, 4, \quad \forall p \in \hat{P}\right\} .
\end{gathered}
$$

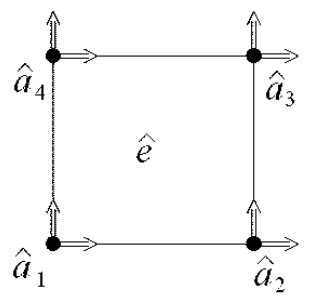

Fig. 1. A double arrow shows the DoF being the value of the second-order derivative it the corresponding direction at the node.

The Lagrange basis for (1) is given by

$$
\begin{array}{ll}
\hat{\varphi}_{0,1}=(1-\hat{x})(1-\hat{y}), & \hat{\varphi}_{0,3}=\hat{x} \hat{y}, \\
\hat{\varphi}_{1,1}=\hat{x}(1-\hat{x})(\hat{x}-2)(1-\hat{y}) / 6, & \hat{\varphi}_{1,3}=\hat{x}\left(\hat{x}^{2}-1\right) \hat{y} / 6, \\
\hat{\varphi}_{2,1}=(1-\hat{x}) \hat{y}(1-\hat{y})(\hat{y}-2) / 6, & \hat{\varphi}_{2,3}=\hat{x} \hat{y}\left(\hat{y}^{2}-1\right) / 6, \\
\hat{\varphi}_{0,2}=\hat{x}(1-\hat{y}), & \hat{\varphi}_{0,4}=(1-\hat{x}) \hat{y}, \\
\hat{\varphi}_{1,2}=\hat{x}\left(\hat{x}^{2}-1\right)(1-\hat{y}) / 6, & \hat{\varphi}_{1,4}=\hat{x}(1-\hat{x})(\hat{x}-2) \hat{y} / 6, \\
\hat{\varphi}_{2,2}=\hat{x} \hat{y}(1-\hat{y})(\hat{y}-2) / 6, & \hat{\varphi}_{2,4}=(1-\hat{x}) \hat{y}\left(\hat{y}^{2}-1\right) / 6 .
\end{array}
$$

For the partition $\mathcal{T}_{h}$, the usual affine mapping of the "reference" element onto an elementary cell $\left[x_{i}, x_{i}+h_{1}\right] \times\left[y_{j}, y_{j}+h_{2}\right]$ is of the form

$$
x=x_{i}+h_{1} \hat{x}, \quad y=y_{j}+h_{2} \hat{y} .
$$

Since $\hat{P} \supset P_{3}$, for the interpolant $\tilde{u}_{I}$ of a function $u \in H^{4}(\Omega)$ on the triangulation $\mathcal{T}_{h}$ we have the usual accuracy estimates $[3,2]$

$$
\begin{gathered}
\left|u-\tilde{u}_{I}\right|_{0, \Omega} \leq c h^{4}\|u\|_{4, \Omega}, \\
\left\|u-\tilde{u}_{I}\right\|_{k, \Omega} \leq c h^{4-k}\|u\|_{4, \Omega}, \quad k=1,2 .
\end{gathered}
$$

From here on, $c$ denotes a positive constant independent of $u$ and $h$.

A feature of element (1) is that for some partial differential equations we can use the collocation technique requiring the residual to vanish at the nodes of 
the elements. This results in considerably reducing the number of unknowns in the system of linear algebraic equations of the finite element method. In [4] this approach is applied to the Poisson equation with numerical results confirming its efficiency.

\section{A Discrete Problem for the Diffusion Equation}

Without loss of generality, for simplicity sake we assume that $\Omega=(0,1) \times(0,1)$. Consider the problem

$$
\begin{gathered}
-\Delta u+k u=f \text { in } \Omega, \\
u=0 \text { on } \Gamma,
\end{gathered}
$$

where $\Delta=\partial^{2} / \partial x^{2}+\partial^{2} / \partial y^{2}$ is the Laplacian, $f \in H^{2}(\Omega), k=$ const $\geq c>0$.

For (5)-(6) we construct the standard scheme of the finite element method with Hermite element (1). Notice that when constructing the partition of $\Omega$, we have $N_{1}=N_{2}=N, h_{1}=h_{2}=h$. We look for an approximate solution in the form

$$
\begin{aligned}
\tilde{u}^{h} & =\sum_{i, j=1}^{N-1} \tilde{u}^{h}\left(z_{i, j}\right) \varphi_{0, i, j}(x, y)+ \\
& +h^{2} \sum_{i, j=0}^{N}\left(\tilde{u}_{x x}^{h}\left(z_{i, j}\right) \varphi_{1, i, j}(x, y)+\tilde{u}_{y y}^{h}\left(z_{i, j}\right) \varphi_{2, i, j}(x, y)\right)
\end{aligned}
$$

where $z_{i, j}=\left(x_{i}, y_{j}\right), x_{i}=i h, y_{j}=j h$; the unknowns $\tilde{u}^{h}\left(z_{i, j}\right), \tilde{u}_{x x}^{h}\left(z_{i, j}\right)$, and $\tilde{u}_{y y}^{h}\left(z_{i, j}\right)$ are the values of the approximate solution and the approximate values of the second-order derivatives of the solution of (5)-(6) at the grid nodes, respectively. The functions $\varphi_{s, i, j}$ form a basis of the finite element space. They are obtained from basis functions (2) of the "reference" element with the affine mapping of the form (3).

As a result, we get a system of linear algebraic equations. The number of equations (and the number of unknowns) equals $3 N_{\text {int }}$ where $N_{\text {int }}$ is the number of interior nodes. The values of $\tilde{u}^{h}, \tilde{u}_{x x}^{h}$, and $\tilde{u}_{y y}^{h}$ at the boundary nodes are determined from (5)-(6).

For convenience sake, we introduce some notations. Consider the grid expression

$$
\sum_{l=j-1}^{j+1} \sum_{k=i-1}^{i+1} a_{k, l} g^{h}\left(z_{k, l}\right)
$$

where $a_{k, l}$ are coefficients and $g^{h}\left(z_{k, l}\right)$ is a grid function. Introduce the nine-point stencil

$$
[A]=\left[\begin{array}{lll}
a_{i-1, j+1} & a_{i, j+1} & a_{i+1, j+1} \\
a_{i-1, j} & a_{i, j} & a_{i+1, j} \\
a_{i-1, j-1} & a_{i, j-1} & a_{i+1, j-1}
\end{array}\right]
$$




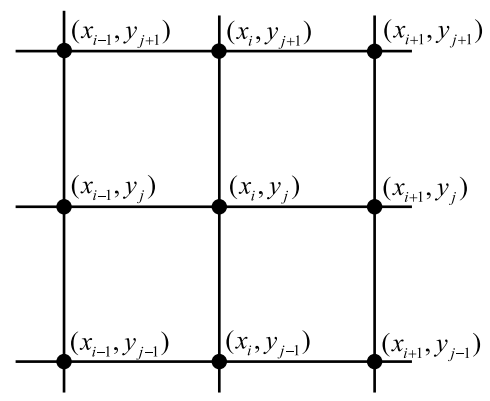

Fig. 2. The nodes of nine-point stencil (7).

where the coefficients $a_{k, l}$ are arranged according to the position of the related nodes (Fig. 2).

Put

$$
[A] g^{h}=\sum_{l=j-1}^{j+1} \sum_{k=i-1}^{i+1} a_{k, l} g^{h}\left(z_{k, l}\right) .
$$

Besides, we use the notation $\left[A^{T}\right]$ for the stencil with the coefficients $a_{k, l}^{T}=a_{l, k}$.

Introduce the grid functions $u^{h}, u_{x x}^{h}$, and $u_{y y}^{h}$ defined by $u^{h}\left(z_{i, j}\right)=\tilde{u}^{h}\left(z_{i, j}\right)$, $u_{x x}^{h}\left(z_{i, j}\right)=\tilde{u}_{x x}^{h}\left(z_{i, j}\right)$, and $u_{y y}^{h}\left(z_{i, j}\right)=\tilde{u}_{y y}^{h}\left(z_{i, j}\right)$. Calculating directly the entries of the stiffness matrix, we arrive at the grid equations of three types

$$
\begin{aligned}
\left(\left[A_{0}\right]\right. & \left.+k h^{2}\left[A_{1}\right]\right) u^{h}+\left(\left[A_{2}\right]+k h^{2}\left[A_{4}\right]\right) h^{2} u_{x x}^{h}+ \\
& +\left(\left[A_{2}^{T}\right]+k h^{2}\left[A_{4}^{T}\right]\right) h^{2} u_{y y}^{h}=\int_{\Omega} f \varphi_{0, i, j} d \Omega \\
\left(\left[A_{2}\right]\right. & \left.+k h^{2}\left[A_{4}\right]\right) u^{h}+\left(\left[A_{3}\right]-\left[A_{4}\right]+k h^{2}\left[A_{5}\right]\right) h^{2} u_{x x}^{h}+ \\
& +k h^{2}\left[A_{6}\right] h^{2} u_{y y}^{h}=\int_{\Omega} f \varphi_{1, i, j} d \Omega, \\
\left(\left[A_{2}^{T}\right]+k h^{2}\left[A_{4}^{T}\right]\right) u^{h}+k h^{2}\left[A_{6}^{T}\right] h^{2} u_{x x}^{h}+ & +\left(\left[A_{3}^{T}\right]-\left[A_{4}^{T}\right]+k h^{2}\left[A_{5}^{T}\right]\right) h^{2} u_{y y}^{h}=\int_{\Omega} f \varphi_{2, i, j} d \Omega,
\end{aligned}
$$

for all $i, j=1, \ldots, N-1$ with the stencils

$$
\begin{gathered}
{\left[A_{0}\right]=\left[\begin{array}{rr}
-1 / 3 & -1 / 3-1 / 3 \\
-1 / 3 & 8 / 3-1 / 3 \\
-1 / 3 & -1 / 3-1 / 3
\end{array}\right], \quad\left[A_{1}\right]=\left[\begin{array}{rrr}
1 / 36 & 1 / 9 & 1 / 36 \\
1 / 9 & 4 / 9 & 1 / 9 \\
1 / 36 & 1 / 9 & 1 / 36
\end{array}\right],} \\
{\left[A_{2}\right]=\left[\begin{array}{rrr}
7 / 360 & 2 / 45 & 7 / 360 \\
-7 / 180 & -4 / 45 & -7 / 180 \\
7 / 360 & 2 / 45 & 7 / 360
\end{array}\right], \quad\left[A_{3}\right]=\left[\begin{array}{rrr}
-31 / 15120 & -4 / 945 & -31 / 15120 \\
31 / 7560 & 8 / 945 & 31 / 7560 \\
-31 / 15120 & -4 / 945 & 31 / 15120
\end{array}\right],}
\end{gathered}
$$




$$
\begin{aligned}
& {\left[A_{4}\right]=\left[\begin{array}{rr}
-7 / 2160-1 / 135 & -7 / 2160 \\
-7 / 540-4 / 135 & -7 / 540 \\
-7 / 2160-1 / 135 & -7 / 2160
\end{array}\right],\left[A_{5}\right]=\left[\begin{array}{lll}
31 / 90720 & 2 / 2835 & 31 / 90720 \\
31 / 22680 & 8 / 2835 & 31 / 22680 \\
31 / 90720 & 2 / 2835 & 31 / 90720
\end{array}\right],} \\
& {\left[A_{6}\right]=\left[\begin{array}{rrr}
49 / 129600 & 7 / 8100 & 49 / 129600 \\
7 / 8100 & 4 / 2025 & 7 / 8100 \\
49 / 129600 & 7 / 8100 & 49 / 129600
\end{array}\right] .}
\end{aligned}
$$

At the nodes near the boundary equations (8)-(10) are modified in an obvious way.

Notice that the values of $u_{x x}^{h}$ and $u_{y y}^{h}$ are equipped with the factor $h^{2}$. We use the $\operatorname{DoF} \psi_{0, i, j}\left(\tilde{u}^{h}\right)=\tilde{u}^{h}\left(z_{i, j}\right), \psi_{1, i, j}\left(\tilde{u}^{h}\right)=h^{2} \tilde{u}_{x x}^{h}\left(z_{i, j}\right), \psi_{2, i, j}\left(\tilde{u}^{h}\right)=h^{2} \tilde{u}_{y y}^{h}\left(z_{i, j}\right)$ as the unknowns of system (8)-(10). This is convenient for several reasons, in particular, this improves properties of the stiffness matrix.

\section{The Elimination of Unknowns by Collocation}

The grid functions $u^{h}, u_{x x}^{h}$, and $u_{y y}^{h}$ satisfy the algebraic identity

$$
-u_{x x}^{h}\left(z_{i, j}\right)-u_{y y}^{h}\left(z_{i, j}\right)+k u^{h}\left(z_{i, j}\right)=f^{h}\left(z_{i, j}\right)-r^{h}\left(z_{i, j}\right),
$$

where $r^{h}$ is the residual, $f^{h}$ is a grid function defined by $f^{h}\left(z_{i, j}\right)=f\left(z_{i, j}\right)$.

Assuming the residual is known, neglect one group of equations, for instance, (10); express $u_{y y}^{h}\left(z_{i, j}\right)$ from (11) and substitute it into (8)-(9). As a result, we obtain the following system

$$
\begin{aligned}
\left(\left[A_{0}\right]\right. & \left.+k h^{2}\left(\left[A_{1}\right]+\left[A_{2}^{T}\right]\right)+k^{2} h^{4}\left[A_{4}^{T}\right]\right) u^{h}+ \\
& +\left(\left[A_{2}\right]-\left[A_{2}^{T}\right]+k h^{2}\left(\left[A_{4}\right]-\left[A_{4}^{T}\right]\right)\right) h^{2} u_{x x}^{h}= \\
& =\int_{\Omega} f \varphi_{0, i, j} d \Omega+\left(\left[A_{2}^{T}\right]+k h^{2}\left[A_{4}^{T}\right]\right) h^{2}\left(f^{h}-r^{h}\right), \\
\left(\left[A_{2}\right]\right. & \left.+k h^{2}\left[A_{4}\right]+k^{2} h^{4}\left[A_{6}\right]\right) u^{h}+\left(\left[A_{3}\right]-\left[A_{4}\right]+k h^{2}\left(\left[A_{5}\right]-\left[A_{6}\right]\right)\right) h^{2} u_{x x}^{h}= \\
& =\int_{\Omega} f \varphi_{1, i, j} d \Omega+k h^{4}\left[A_{6}\right]\left(f^{h}-r^{h}\right),
\end{aligned}
$$

for all $i, j=1, \ldots, N-1$, reducing the number of equations by a factor of $1 / 3$.

Now introduce grid functions $v^{h}, v_{x x}^{h}$, and $v_{y y}^{h}$ being the values of a new approximate solution and new approximate values of the second-order derivatives at the nodes, respectively, and require that the residual vanish at the nodes:

$$
-v_{x x}^{h}\left(z_{i, j}\right)-v_{y y}^{h}\left(z_{i, j}\right)+k v^{h}\left(z_{i, j}\right)=f^{h}\left(z_{i, j}\right) .
$$

In (12)-(13), replace $u^{h}$ and $u_{x x}^{h}$ by $v^{h}$ and $v_{x x}^{h}$, respectively, and put $r^{h}=0$. This gives a system of equations for the new unknowns. Once the system has been solved, $v_{y y}^{h}\left(z_{i, j}\right)$ can readily be determined from (14). 
We are interested in accuracy of the new approximate solution. In [4] we use finite element (1) coupled with collocation for the Poisson equation. Numerical experiments show that a solution on the reduced system is of the same order of accuracy as that of the original system of the finite element method. Below we prove this result.

\section{An Error Estimate}

Consider an arbitrary function

$$
\tilde{w}=\sum_{i, j=1}^{N-1} w_{i, j} \varphi_{0, i, j}(x, y)+h^{2} \sum_{i, j=0}^{N}\left(\left(w_{x x}\right)_{i, j} \varphi_{1, i, j}(x, y)+\left(w_{y y}\right)_{i, j} \varphi_{2, i, j}(x, y)\right)
$$

of the finite element space. To this function, there corresponds the vector of the coefficients

$$
W=\left(\left\{w_{i, j}\right\}_{i, j=1}^{N-1},\left\{h^{2}\left(w_{x x}\right)_{i, j}\right\}_{i, j=0}^{N},\left\{h^{2}\left(w_{y y}\right)_{i, j}\right\}_{i, j=0}^{N}\right)
$$

of a linear vector space $M$. The function $\tilde{w}$ is called a prolongation of the vector $W$.

In the vector space $M$ we introduce the weighted Euclidean norm

$$
\|W\|=\left(\sum_{i, j=1}^{N-1} w_{i, j}^{2}+h^{4}\left(\sum_{i, j=0}^{N}\left(\left(w_{x x}\right)_{i, j}^{2}+\left(w_{y y}\right)_{i, j}^{2}\right)\right)\right)^{1 / 2} .
$$

It is equivalent to the norm $|\tilde{w}|_{0, \Omega}$ of the prolongation up to the factor $h[7]$ :

$$
c^{*} h\|W\| \leq|\tilde{w}|_{0, \Omega} \leq c^{* *} h\|W\|
$$

Denote the prolongation of the vector

$$
V^{h}=\left(\left\{v^{h}\left(z_{i, j}\right)\right\}_{i, j=1}^{N-1}, \quad\left\{h^{2} v_{x x}^{h}\left(z_{i, j}\right)\right\}_{i, j=0}^{N}, \quad\left\{h^{2} v_{y y}^{h}\left(z_{i, j}\right)\right\}_{i, j=0}^{N}\right)
$$

by $\tilde{v}^{h}$.

Theorem 1. An approximate solution $\tilde{v}^{h}$ of problem (5)-(6) satisfies the estimate

$$
\left|u-\tilde{v}^{h}\right|_{0, \Omega} \leq \operatorname{ch}^{4}\|u\|_{4, \Omega} .
$$

Proof. We have system (12)-(13) for the unknowns $u^{h}$ and $h^{2} u_{x x}^{h}$ and the system with the same matrix for the unknowns $v^{h}$ and $h^{2} v_{x x}^{h}$. The only difference between these systems is in the terms

$$
\delta^{0, h}=\left(\left[A_{2}^{T}\right]+k h^{2}\left[A_{4}^{T}\right]\right) h^{2} r^{h},
$$




$$
\delta^{1, h}=k h^{4}\left[A_{6}\right] r^{h}
$$

in the right-hand side.

Introduce the vectors

$$
\begin{gathered}
U^{h}=\left(\left\{u^{h}\left(z_{i, j}\right)\right\}_{i, j=1}^{N-1},\left\{h^{2} u_{x x}^{h}\left(z_{i, j}\right)\right\}_{i, j=0}^{N},\left\{h^{2} u_{y y}^{h}\left(z_{i, j}\right)\right\}_{i, j=0}^{N}\right), \\
\bar{U}^{h}=\left(\left\{u^{h}\left(z_{i, j}\right)\right\}_{i, j=1}^{N-1},\left\{h^{2} u_{x x}^{h}\left(z_{i, j}\right)\right\}_{i, j=0}^{N}\right) \\
\bar{V}^{h}=\left(\left\{v^{h}\left(z_{i, j}\right)\right\}_{i, j=1}^{N-1},\left\{h^{2} v_{x x}^{h}\left(z_{i, j}\right)\right\}_{i, j=0}^{N}\right) .
\end{gathered}
$$

The matrix of system (12)-(13) is equivalent to the matrix of finite element system with less number of DoF which are reduced by equality (11). Therefore it has the same property of stability. Thus, taking into account (17)-(18), we have the estimate [6]

$$
\left\|\bar{U}^{h}-\bar{V}^{h}\right\| \leq c h^{2}\left\|r^{h}\right\| .
$$

All vector norms are the Euclidean norms in the vector spaces of corresponding dimension.

Now we estimate $\left\|U^{h}-V^{h}\right\|$. Using (11), (14), and the algebraic inequality $(a+b+c)^{2} \leq 4\left(a^{2}+b^{2}+c^{2}\right)$, we get

$$
\begin{aligned}
\| U^{h} & -V^{h} \|^{2}=\sum_{i, j=1}^{N-1}\left(u^{h}\left(z_{i, j}\right)-v^{h}\left(z_{i, j}\right)\right)^{2} \\
& +\sum_{i, j=0}^{N}\left(h^{4}\left(u_{x x}^{h}\left(z_{i, j}\right)-v_{x x}^{h}\left(z_{i, j}\right)\right)^{2}\right. \\
& \left.+h^{4}\left(k\left(u^{h}\left(z_{i, j}\right)-v^{h}\left(z_{i, j}\right)\right)+v_{x x}^{h}\left(z_{i, j}\right)-u_{x x}^{h}\left(z_{i, j}\right)+r^{h}\left(z_{i, j}\right)\right)^{2}\right) \\
& \leq c\left(\sum_{i, j=1}^{N-1}\left(u^{h}\left(z_{i, j}\right)-v^{h}\left(z_{i, j}\right)\right)^{2}+h^{4} \sum_{i, j=0}^{N}\left(u_{x x}^{h}\left(z_{i, j}\right)-v_{x x}^{h}\left(z_{i, j}\right)\right)^{2}\right) \\
& +c h^{4} \sum_{i, j=0}^{N}\left(r^{h}\left(z_{i, j}\right)\right)^{2}=c\left\|\bar{U}^{h}-\bar{V}^{h}\right\|^{2}+c h^{4}\left\|r^{h}\right\|^{2} .
\end{aligned}
$$

Combining this inequality with (19) gives

$$
\left\|U^{h}-V^{h}\right\| \leq c h^{2}\left\|r^{h}\right\| .
$$

From (5) and (11) we have

$$
r^{h}\left(z_{i, j}\right)=u_{x x}^{h}\left(z_{i, j}\right)-\frac{\partial^{2} u}{\partial x^{2}}\left(z_{i, j}\right)+u_{y y}^{h}\left(z_{i, j}\right)-\frac{\partial^{2} u}{\partial y^{2}}\left(z_{i, j}\right)+k\left(u\left(z_{i, j}\right)-u^{h}\left(z_{i, j}\right)\right) .
$$


Hence,

$$
\begin{aligned}
\left\|r^{h}\right\|^{2} \leq & c\left(\sum_{i, j=1}^{N-1}\left(u^{h}\left(z_{i, j}\right)-u\left(z_{i, j}\right)\right)^{2}+\sum_{i, j=0}^{N}\left(\left(u_{x x}^{h}\left(z_{i, j}\right)-\frac{\partial^{2} u}{\partial x^{2}}\left(z_{i, j}\right)\right)^{2}\right.\right. \\
& \left.\left.+\left(u_{y y}^{h}\left(z_{i, j}\right)-\frac{\partial^{2} u}{\partial y^{2}}\left(z_{i, j}\right)\right)^{2}\right)\right) \\
\leq c h^{-4} & \left(\sum_{i, j=1}^{N-1}\left(u^{h}\left(z_{i, j}\right)-u\left(z_{i, j}\right)\right)^{2}+\sum_{i, j=0}^{N}\left(h^{4}\left(u_{x x}^{h}\left(z_{i, j}\right)-\frac{\partial^{2} u}{\partial x^{2}}\left(z_{i, j}\right)\right)^{2}\right.\right. \\
& \left.\left.+h^{4}\left(u_{y y}^{h}\left(z_{i, j}\right)-\frac{\partial^{2} u}{\partial y^{2}}\left(z_{i, j}\right)\right)^{2}\right)\right)=c h^{-4}\left\|U^{h}-U_{I}\right\|^{2}
\end{aligned}
$$

where the vector $U_{I}$ consists of the coefficients of the interpolant $\tilde{u}_{I}$. Taking into account norm equivalence (15), we obtain

$$
\left\|r^{h}\right\| \leq c h^{-3}\left|\tilde{u}^{h}-\tilde{u}_{I}\right|_{0, \Omega} .
$$

For the interpolant $\tilde{u}_{I}$ we have estimate (4). Besides, the approximate solution $\tilde{u}^{h}$ satisfies the estimate [4]

$$
\left|u-\tilde{u}^{h}\right|_{0, \Omega} \leq c h^{4}\|u\|_{4, \Omega} .
$$

Hence, with the help of the triangle inequality from (21) we get

$$
\left\|r^{h}\right\| \leq c^{-3}\left(\left|u-\tilde{u}^{h}\right|_{0, \Omega}+\left|u-\tilde{u}_{I}\right|_{0, \Omega}\right) \leq c h\|u\|_{4, \Omega} .
$$

Finally, from (20), (15), and (23) we conclude that

$$
\left|\tilde{u}^{h}-\tilde{v}^{h}\right|_{0, \Omega} \leq c h\left\|U^{h}-V^{h}\right\| \leq \operatorname{ch}^{4}\|u\|_{4, \Omega},
$$

which together with (22) proves the theorem.

\section{Numerical Results}

Let $\Omega=(0,1) \times(0,1)$ and $\Gamma$ be the boundary of $\Omega$. Consider the boundary value problem

$$
\begin{aligned}
& -\Delta u+0.5 u=f \quad \text { in } \Omega, \\
& u=\left\{\begin{array}{lr}
0 & \text { for } x=0, \\
0 & \text { for } y=0, \\
-10000 y \sin y & \text { for } x=1, \\
-10000 y \sin x & \text { for } y=1
\end{array}\right.
\end{aligned}
$$

with the right-hand side

$$
f(x, y)=5000(4(x+y) \cos (1-x-y)+5 x y \sin (1-x-y)) .
$$


The exact solution of (24)-(26) has the form

$$
u(x, y)=10000 x y \sin (1-x-y) .
$$

To construct a discrete problem, we subdivide $\Omega$ into elementary squares for mesh size $h=1 / N$. In numerical experiments we use $N=5,10,20,40,80$. On the obtained partition, along with the standard system of the finite element method for Hermite element (1), we construct and solve the modified system of smaller dimension obtained with the help of collocation.

Since exact solution (27) is known, the error $u\left(z_{i, j}\right)-u^{h}\left(z_{i, j}\right)$ or $u\left(z_{i, j}\right)-v^{h}\left(z_{i, j}\right)$ of an approximate solution at the node $z_{i, j}$ can be calculated explicitly. Besides, we can calculate $\partial^{2} u / \partial x^{2}\left(z_{i, j}\right)-u_{x x}^{h}\left(z_{i, j}\right)$ and $\partial^{2} u / \partial y^{2}\left(z_{i, j}\right)-u_{y y}^{h}\left(z_{i, j}\right)$ or $\partial^{2} u / \partial x^{2}\left(z_{i, j}\right)-v_{x x}^{h}\left(z_{i, j}\right)$ and $\partial^{2} u / \partial y^{2}\left(z_{i, j}\right)-v_{y y}^{h}\left(z_{i, j}\right)$ to estimate convergence of the second-order derivatives. We use the discrete norms

$$
\begin{gathered}
\left\|u^{h}\right\|_{h}=\left(\sum_{i, j=1}^{N-1}\left(u^{h}\left(z_{i, j}\right)\right)^{2} h^{2}\right)^{1 / 2}, \\
\left\|u-u^{h}\right\|_{h}=\left(\sum_{i, j=1}^{N-1}\left(u\left(z_{i, j}\right)-u^{h}\left(z_{i, j}\right)\right)^{2} h^{2}\right)^{1 / 2} .
\end{gathered}
$$

In addition, we also use the following discrete norm for a relative error:

$$
\left\|u-u^{h}\right\|_{h}^{r e l}=\frac{\left\|u-u^{h}\right\|_{h}}{\left\|u^{h}\right\|_{h}} .
$$

Table 1. Accuracy of the approximate solution for the standard system.

\begin{tabular}{|r|c|c|c|c|}
\hline \multicolumn{1}{|c|}{$h$} & $\varepsilon^{h}\left(u^{h}\right)$ & $\sigma_{x}^{h}\left(u^{h}\right)$ & $\sigma_{y}^{h}\left(u^{h}\right)$ & $\sigma_{\Delta}^{h}\left(u^{h}\right)$ \\
\hline 0.2 & $4.22 E-1$ & $1.46 E+2$ & $1.46 E+2$ & $2.86 E+2$ \\
0.1 & $2.97 E-2$ & $3.90 E+1$ & $3.90 E+1$ & $7.60 E+1$ \\
0.05 & $1.97 E-3$ & $9.93 E+0$ & $9.93 E+0$ & $1.94 E+1$ \\
0.025 & $1.27 E-4$ & $2.51 E+0$ & $2.51 E+0$ & $4.91 E+0$ \\
0.0125 & $7.17 E-6$ & $6.30 E-1$ & $6.30 E-1$ & $1.24 E+0$ \\
\hline
\end{tabular}

In Tab. 1-3 we present results of calculations for the standard system of the finite element method. We use the following notations:

$$
\begin{array}{clrl}
\varepsilon^{h}\left(u^{h}\right)=\left\|u-u^{h}\right\|_{h}, & \bar{\varepsilon}^{h}\left(u^{h}\right)=\left\|u-u^{h}\right\|_{h}^{r e l}, \\
\sigma_{x}^{h}\left(u^{h}\right)=\left\|\frac{\partial^{2} u}{\partial x^{2}}-u_{x x}^{h}\right\|_{h}, & \bar{\sigma}_{x}^{h}\left(u^{h}\right)=\left\|\frac{\partial^{2} u}{\partial x^{2}}-v_{x x}^{h}\right\|_{h}^{r e l}, \\
\sigma_{y}^{h}\left(u^{h}\right)=\left\|\frac{\partial^{2} u}{\partial y^{2}}-u_{y y}^{h}\right\|_{h}, & \bar{\sigma}_{y}^{h}\left(u^{h}\right)=\left\|\frac{\partial^{2} u}{\partial y^{2}}-v_{y y}^{h}\right\|_{h}^{r e l},
\end{array}
$$


Table 2. Relative error for the standard system.

\begin{tabular}{|r|c|c|c|c|}
\hline \multicolumn{1}{|c|}{$h$} & $\bar{\varepsilon}^{h}\left(u^{h}\right)$ & $\bar{\sigma}_{x}^{h}\left(u^{h}\right)$ & $\bar{\sigma}_{y}^{h}\left(u^{h}\right)$ & $\bar{\sigma}_{\Delta}^{h}\left(u^{h}\right)$ \\
\hline 0.2 & $1.86 \mathrm{E}-4$ & $1.50 \mathrm{E}-2$ & $1.50 \mathrm{E}-2$ & $1.68 \mathrm{E}-2$ \\
0.1 & $1.47 \mathrm{E}-5$ & $4.02 \mathrm{E}-3$ & $4.02 \mathrm{E}-3$ & $4.38 \mathrm{E}-3$ \\
0.05 & $1.05 \mathrm{E}-6$ & $1.02 \mathrm{E}-3$ & $1.02 \mathrm{E}-3$ & $1.11 \mathrm{E}-3$ \\
0.025 & $6.99 \mathrm{E}-8$ & $2.58 \mathrm{E}-4$ & $2.58 \mathrm{E}-4$ & $2.78 \mathrm{E}-4$ \\
0.0125 & $4.03 \mathrm{E}-9$ & $6.49 \mathrm{E}-5$ & $6.49 \mathrm{E}-5$ & $6.98 \mathrm{E}-5$ \\
\hline
\end{tabular}

Table 3. A numerical convergence estimate for the standard system.

\begin{tabular}{|r|c|c|c|c|}
\hline$h$ & $\log \left(\frac{\varepsilon^{h}\left(u^{2 h}\right)}{\varepsilon^{h}\left(u^{h}\right)}\right)$ & $\log \left(\frac{\sigma_{x}^{h}\left(u^{2 h}\right)}{\sigma_{x}^{h}\left(u^{h}\right)}\right)$ & $\log \left(\frac{\sigma_{y}^{h}\left(u^{2 h}\right)}{\sigma_{y}^{h}\left(u^{h}\right)}\right)$ & $\log \left(\frac{\sigma_{\Delta}^{h}\left(u^{2 h}\right)}{\sigma_{\Delta}^{h}\left(u^{h}\right)}\right)$ \\
\hline 0.1 & 3.8 & 1.9 & 1.9 & 1.9 \\
0.05 & 3.9 & 2.0 & 2.0 & 2.0 \\
0.025 & 4.0 & 2.0 & 2.0 & 2.0 \\
0.0125 & 4.1 & 2.0 & 2.0 & 2.0 \\
\hline
\end{tabular}

$$
\sigma_{\Delta}^{h}\left(u^{h}\right)=\left\|\Delta u-\Delta u^{h}\right\|_{h}, \quad \bar{\sigma}_{\Delta}^{h}\left(u^{h}\right)=\left\|\Delta u-\Delta u^{h}\right\|_{h}^{r e l},
$$

where $\Delta u^{h}\left(z_{i, j}\right)=u_{x x}^{h}\left(z_{i, j}\right)+u_{y y}^{h}\left(z_{i, j}\right)$ is an approximate value of $\Delta u$ at the node.

Observe that the error $\varepsilon^{h}\left(u^{h}\right)$ tends to be of order 4 as $h$ decreases while $\sigma_{x}^{h}\left(u^{h}\right)$ and $\sigma_{y}^{h}\left(u^{h}\right)$ tend to be of order 2. This is in good agreement with theoretical estimate $(22)$.

Another series of calculations is related to the solution of the reduced system for the unknowns $v^{h}\left(z_{i, j}\right)$ and $v_{x x}^{h}\left(z_{i, j}\right)$. In this case the values of $v_{y y}^{h}$ are determined from (14). Besides, from (14) we also directly determine the approximate values of $\Delta u$ at the nodes:

$$
\Delta v^{h}\left(z_{i, j}\right)=k v^{h}\left(z_{i, j}\right)-f^{h}\left(z_{i, j}\right)
$$

The results of calculations are presented in Tab. 4-6.

Table 4. Accuracy of the approximate solution for the reduced system.

\begin{tabular}{|r|c|c|c|c|}
\hline \multicolumn{1}{|c|}{$h$} & $\varepsilon^{h}\left(v^{h}\right)$ & $\sigma_{x}^{h}\left(v^{h}\right)$ & $\sigma_{y}^{h}\left(v^{h}\right)$ & $\sigma_{\Delta}^{h}\left(v^{h}\right)$ \\
\hline 0.2 & $2.45 \mathrm{E}-1$ & $1.39 \mathrm{E}+2$ & $1.39 \mathrm{E}+2$ & $1.22 \mathrm{E}-1$ \\
0.1 & $1.87 \mathrm{E}-2$ & $3.84 \mathrm{E}+1$ & $3.84 \mathrm{E}+1$ & $9.34 \mathrm{E}-3$ \\
0.05 & $1.21 \mathrm{E}-3$ & $9.88 \mathrm{E}+0$ & $9.88 \mathrm{E}+0$ & $6.04 \mathrm{E}-4$ \\
0.025 & $7.59 \mathrm{E}-5$ & $2.50 \mathrm{E}+0$ & $2.50 \mathrm{E}+0$ & $3.79 \mathrm{E}-5$ \\
0.0125 & $5.18 \mathrm{E}-6$ & $6.30 \mathrm{E}-1$ & $6.30 \mathrm{E}-1$ & $2.59 \mathrm{E}-6$ \\
\hline
\end{tabular}

Notice that the errors $\varepsilon^{h}\left(v^{h}\right), \sigma_{x}^{h}\left(v^{h}\right)$, and $\sigma_{y}^{h}\left(v^{h}\right)$ are close to those for $u^{h}$ which confirms estimate (16). Moreover, in the case of the reduced system the 
Table 5. Relative error for the reduced system.

\begin{tabular}{|r|c|c|c|c|}
\hline \multicolumn{1}{c|}{$h$} & $\bar{\varepsilon}^{h}\left(v^{h}\right)$ & $\bar{\sigma}_{x}^{h}\left(v^{h}\right)$ & $\bar{\sigma}_{y}^{h}\left(v^{h}\right)$ & $\bar{\sigma}_{\Delta}^{h}\left(v^{h}\right)$ \\
\hline 0.2 & $1.07 \mathrm{E}-4$ & $1.44 \mathrm{E}-2$ & $1.44 \mathrm{E}-2$ & $7.20 \mathrm{E}-6$ \\
0.1 & $9.28 \mathrm{E}-6$ & $3.96 \mathrm{E}-3$ & $3.96 \mathrm{E}-3$ & $5.38 \mathrm{E}-7$ \\
0.05 & $6.42 \mathrm{E}-7$ & $1.02 \mathrm{E}-3$ & $1.02 \mathrm{E}-3$ & $3.44 \mathrm{E}-8$ \\
0.025 & $4.19 \mathrm{E}-8$ & $2.58 \mathrm{E}-4$ & $2.58 \mathrm{E}-4$ & $2.15 \mathrm{E}-9$ \\
0.0125 & $2.92 \mathrm{E}-9$ & $6.49 \mathrm{E}-5$ & $6.49 \mathrm{E}-5$ & $1.46 \mathrm{E}-10$ \\
\hline
\end{tabular}

Table 6. A numerical convergence estimate for the reduced system.

\begin{tabular}{|r|c|c|c|c|}
\hline$h$ & $\log \left(\frac{\varepsilon^{h}\left(v^{2 h}\right)}{\varepsilon^{h}\left(v^{h}\right)}\right)$ & $\log \left(\frac{\sigma_{x}^{h}\left(v^{2 h}\right)}{\sigma_{x}^{h}\left(v^{h}\right)}\right)$ & $\log \left(\frac{\sigma_{y}^{h}\left(v^{2 h}\right)}{\sigma_{y}^{h}\left(v^{h}\right)}\right)$ & $\log \left(\frac{\sigma_{\Delta}^{h}\left(v^{2 h}\right)}{\sigma_{\Delta}^{h}\left(v^{h}\right)}\right)$ \\
\hline 0.1 & 3.7 & 1.9 & 1.9 & 3.7 \\
0.05 & 4.0 & 2.0 & 2.0 & 4.0 \\
0.025 & 4.0 & 2.0 & 2.0 & 4.0 \\
0.0125 & 3.9 & 2.0 & 2.0 & 3.9 \\
\hline
\end{tabular}

accuracy is even slightly better. In addition, from (28) we can calculate approximate values of $\Delta u$ within $O\left(h^{4}\right)$.

Thus, numerical experiments confirm the theoretical estimate and demonstrate the advantage of the proposed approach.

\section{References}

1. Adams, R., Fournier, I.: Sobolev Spaces. Academic Press, NY, USA (2003)

2. Brenner, S., Scott, L.: The Mathematical Theory of Finite Element Method. Springer-Verlag, Berlin-Heidelberg-New York (1994)

3. Ciarlet, P.: The Finite Element Method for Elliptic Problems. North Holland, Amsterdam, Netherlands (1978)

4. Gileva, L., Karepova, E., Shaidurov, V.: New hermite finite elements on rectangles. In: Todorov, M.D. (ed.) Application of Mathematics In Technical and Natural Sciences: 8th International Conference for Promoting the Application of Mathematics in Technical and Natural Sciences - AMiTaNS16. AIP Conference Proceedings, vol. 1773, pp. 100005-1-100005-7. AIP Publishing, NY, USA (2016)

5. Gileva, L., Shaydurov, V.: Bicubic hermite elements in a domain with the curved boundary. Lobachevskii Journal of Mathematics 39(7), 893-903 (2018)

6. Samarskii, A.: The Theory of Difference Schemes. CRC Press, USA (2001)

7. Shaidurov, V.: Multigrid Method for Finite Elements. Kluwer Academic Publishers, Netherlands (1995)

8. Shaidurov, V., Shut, S., Gileva, L.: Some properties of hermite finite elements on rectangles. In: Todorov, M.D. (ed.) Application of Mathematics In Technical and Natural Sciences: 6th International Conference for Promoting the Application of Mathematics in Technical and Natural Sciences AMiTaNS 14. AIP Conference Proceedings, vol. 1629, pp. 32-43. AIP Publishing, NY, USA (2014) 